This document is the accepted manuscript version of the following article:

Chen, M., Best, J. P., Shorubalko, I., Michler, J., Spolenak, R., \& wheeler, J. M. (2020). Influence of helium ion irradiation on the structure and strength of diamond. Carbon, 158, 337-345. https://doi.org/10.1016/j.carbon.2019.10.078

This manuscript version is made available under the CC-BY-NC-ND 4.0 license http://creativecommons.org/1icenses/by-nc-nd/4.0/

\title{
Influence of helium ion irradiation on the structure and strength of diamond
}

Ming Chen ${ }^{1}$, James P. Best ${ }^{2,3}$, Ivan Shorubalko ${ }^{4}$, Johann Michler ${ }^{2}$, Ralph Spolenak ${ }^{1}$ and Jeffrey M. Wheeler ${ }^{1 *}$

${ }^{1}$ Laboratory for Nanometallurgy, ETH Zürich, Vladimir-Prelog-Weg 5, CH-8093 Zürich, Switzerland

2 Laboratory for Mechanics of Materials and Nanostructures, Empa, Swiss Federal Laboratories for Materials Science and Technology, Feuerwerkerstrasse 39, CH-3602 Thun, Switzerland

${ }^{3}$ School of Mechanical and Manufacturing Engineering, UNSW Sydney, NSW 2052, Australia

${ }^{4}$ Laboratory for Transport at Nanoscale Interfaces, Empa, Swiss Federal Laboratories for Materials Science and Technology, Ueberlandstrasse 129, CH-8600 Dübendorf, Switzerland

KEYWORDS : Diamond; Crystalline orientation; Micro-mechanics; Helium-ionmicroscope; ion channelling

*Corresponding author. Tel: +41 4463328 63. E-mail: jeff.wheeler@mat.ethz.ch 
ABSTRACT: Microfabrication of synthetic single crystal diamond using accelerated helium ions beams has significant potential for functional applications such as high precision optical devices, through tailoring of the optical properties via diamond graphitization. The use of helium ion microscopes (HIM) with nano-scaled focused ion beam spot sizes also allows for precision nano-patterning of the diamond surface through post-exposure selective etching of the generated graphitic phase. Here, single-crystalline diamonds with $\langle 100\rangle,\langle 110\rangle,\langle 111\rangle$ and $\langle 123\rangle$ orientations are exposed to $\mathrm{He}$ ion radiation from HIM, at a range of acceleration voltages and fluences. It is observed that $\langle 123\rangle$ orientation was more sensitive to ion irradiation as notably $\mathrm{sp}^{3}$ to $\mathrm{sp}^{2}$ bonds transition at low fluence $\left(10^{15}\right.$ ions $\left./ \mathrm{cm}^{2}\right)$ compared to other orientations. In situ uniaxial compression of SC diamond micro-pillars reveals the strength of $\langle 123\rangle$-oriented pillars is strongly dependent on the ion fluence, and thus is tunable by ion irradiation. Notably, $\langle 100\rangle$-oriented pillars exhibit a better damage resistance as a small strength degradation due to its higher ion channeling efficiency. The irradiation damage of energetic helium ions on structure and strength of diamond is therefore highly orientation-dependent. Such results provide the critical knowledge for precise patterning and designing of diamond-based functional structures in nano-fabrication.

\section{Introduction}

Single-crystalline (SC) diamond is the hardest known material, as well as the material with the highest thermal conductivity [1]. SC diamond is also an excellent electrical insulator for electronic applications [2]. Based on such exceptional properties, diamond is used in many technical applications, especially within the field of precision science such as force and temperature sensing in nanoscales [3-5]. Together with isotropic low scattering optical properties, SC diamond is also an attractive material for demanding optical applications as for wide spectral range windows in laser systems [6]. Presently, precise modification of diamond 
for both fabricating three dimensional structures and tailoring local properties have been a significant topic for myriad applications in functional microstructures and devices [7-9]. As one of the most popular fabrication approaches for the nanostructured materials, ion beam machining affords high spatial resolution and geometrical flexibility at the micro- and nanoscales [10]. Below the graphitization threshold of ion damage level, defects induced by ion damage can tailor the local optical/electrical properties of the diamond with applications in precision optics, e.g. waveguides $[8,11]$. Above this graphitization threshold, the damaged diamond is subsequently annealed to induce the graphitization, and then selectively etched away. This route has been demonstrably achieved with a good spatial selectivity using both $\mathrm{MeV}$-ion sources and high-power laser devices to pattern diamond and fabricate free-standing structures for applications in small-scale systems $[7,12,13]$. Therefore, accurate knowledge regarding the modification of diamond structure and associated properties after ion radiation is critical for such nanofabrication techniques. In addition, diamond is also envisioned for using as a radiation detector and a particle shield in high energy helium ions irradiation environments such as fusion reactors [14-16]. Thus, it is essential to understand the effect of irradiation damage on the structural and mechanical stabilities of diamond to enable design of robust, diamond-based structures for the nuclear industry.

Currently, graphitization of the diamond structure is achieved using ion micro-beam-lines at $\mathrm{MeV}$ acceleration voltages to raster scan the diamond surface with a beam size of $20-30 \mu \mathrm{m}$ [12]. However, the spatial resolution and fabrication precision are constricted to the scale of the micro-beam, especially in the applications with feature sizes in nanoscales. With recent advances in helium ion microscopy (HIM), a gas field ionization source has been developed for ultra-high resolution scanning; such HIM enables high contrast imaging with an focused ion beam spot size of $\sim 0.25 \mathrm{~nm}$ at $30 \mathrm{keV}$ [17-19]. This new technique has the advantage of extremely small beam sizes both to precisely pattern and to image materials' surfaces. It is 
known that the materials sputtering yield of He ion beam is much lower compared to other ion sources due to the relative small mass and deep penetration of He ions [20]. However, the structural damage and the corresponding properties modification from $\mathrm{He}$ ion exposure on SC diamond have not yet been fully studied with regards to crystalline orientation, ion beam energy and ion fluence.

\section{Experimental}

\subsection{Helium ion irradiation}

In this study, high-pressure high-temperature (HPHT) diamond single crystals with a size of approximately $0.7 \mathrm{~mm}$ were acquired, oriented, and polished into the desired crystallographic orientations (Synton AG, Port, Switzerland) [21]. The diamond surfaces were sputtered with gold nano-islands to minimize the charging effect during microscopic analysis prior to further investigation. Subsequently, the oriented facets of synthetic SC diamond were each exposed to focused $\mathrm{He}$ ion irradiation at fluences in the range of $10^{15}-10^{19} \mathrm{ions} / \mathrm{cm}^{2}$, corresponding to an identical range used for $\mathrm{MeV}$ modification [12]. This was realized by utilizing a commercial HIM (Orion Plus, Carl Zeiss) at a range of acceleration voltages (10-30 $\mathrm{keV})$ to expose a square pattern $\left(3 \times 3 \mu \mathrm{m}^{2}\right)$ on the diamond surfaces. The fluence of each irradiation is equal to the number of implanted helium ions, $N$, divided by the area of irradiation region, $A$, as following:

Fluence $=\frac{N}{A}=\frac{I \cdot t \cdot N_{\text {coulomb }}}{A}$

where $I$ is the current of ion beam, $t$ is the irradiation time and $N_{\text {coulomb }}$ is the charge of one coulomb $\left(6.24 \times 10^{18}\right)$, because the helium ions in HIM are univalent.

\subsection{Raman spectroscopy}


After HIM exposure, confocal Raman spectroscopy analysis was conducted on the center of the irradiated regions in order to understand the underlying damage mechanism. Raman spectroscopy is highly sensitive to covalent bonding and the corresponding structural modification of diamond [22]. A confocal system (NT-MDT, Russia) with a $633 \mathrm{~nm} \mathrm{HeNe}$ excitation source was used for Raman spectroscopy. In this confocal system (objective lens $50 \times$ MPLN, laser power $1 \mathrm{~mW}$ ), the penetration depth of laser light is on the order of $500 \mathrm{~nm}$ beneath the surface, which exactly overlaps the depth of the implanted He ions layer according to the SRIM simulation. Although the absorption coefficient of laser light in the damaged layer is probably increased by $16.6 \%$ after the ion implantation, the decrease of laser penetration depth is still negligible, i.e. tens of nanometers [11].

\subsection{Microcompression tests}

To study the effects of ion irradiation on the resulting mechanical properties, in situ compression of as-fabricated and irradiated diamond micropillars were conducted. Diamond pillars were fabricated using a $\mathrm{Ga}^{+}$focused ion beam (FIB) (Helios G3 station, Thermofischer Scientific) operated at $30 \mathrm{keV}$ of $\mathrm{Ga}^{+}$beam and $5 \mathrm{keV}$ of electron beam sources. Initially, currents of $\sim 700 \mathrm{pA}$ were used to mill craters around pillars with larger than the targeted diameters to allow clear viewing of the entire pillar length during compression. Next, the desired pillar dimensions with $500 \mathrm{~nm}$ top diameters, $1500 \mathrm{~nm}$ height and taper angle $\sim 4^{\circ}$ were achieved by polishing the coarse pillars with lower currents ranging from 200 to $40 \mathrm{pA}$, which in turn minimized radiation damage from $\mathrm{Ga}^{+}$FIB. After that, the diamond nanopillars were irradiated in the HIM under a constant acceleration voltage of $30 \mathrm{keV}$ to various fluences. In the selected orientations, 2 pillars were irradiated to each fluence level to provide samples for individual microcompression testing to determine the influence on strength. The exposed micropillars were then compressed in situ in Zeiss DSM 962 SEM, using an Alemnis SEM Indenter (Alemnis AG, Thun, Switzerland) with a diamond flat punch tip until fracture [23- 
25]. The pillars were compressed at a constant strain rate of $2 \times 10^{-4} \mathrm{~s}^{-1}$ using the intrinsic displacement control mode, and the load-displacement data was recorded. After the correction of test frame compliance and elastic "sink-in" of the pillar substrate, the engineering stressstrain curves from microcompression were obtained by using the top diameters and the total pillar height of the pillars [21].

\section{Results and discussion}

\subsection{Structural damage from He ion irradiation}

The simulations were initially performed to investigate the distribution of implanted $\mathrm{He}$ ions into diamond after the normal incidence bombardment by using a Monte Carlo approach (SRIM-2013.00, http://www.srim.org) [26]. An average He ion penetration depth of $128 \mathrm{~nm}$ was found for 100,000 ion interactions, when using an isotropic diamond density of 3.514 $\mathrm{g} \cdot \mathrm{cm}^{-3}$ and an acceleration voltage of $30 \mathrm{keV}$ with $90^{\circ}$ incidence angle, as shown in Fig. 1a. For the carbon atoms in the diamond, the displacement energy was $28 \mathrm{eV}$, the lattice bonding energy was $6.29 \mathrm{eV}$ and the surface bonding energy was $6.67 \mathrm{eV}[27,28]$.This penetration depth was approximately one order of magnitude greater than gallium ions $(\sim 10-50 \mathrm{~nm})$ at the same acceleration voltage, due to lower energy loss rate according to previous measurements and simulation [29]. The lateral (radial) distribution at the average ion penetration depth was approximately $55 \mathrm{~nm}$. The penetration depth of He ions in diamond is highly dependent on the beam energy, where the average depth of He ions is decreased to $52 \mathrm{~nm}$ at the acceleration voltage of $10 \mathrm{keV}$ shown in Fig. $1 \mathbf{b}$. 

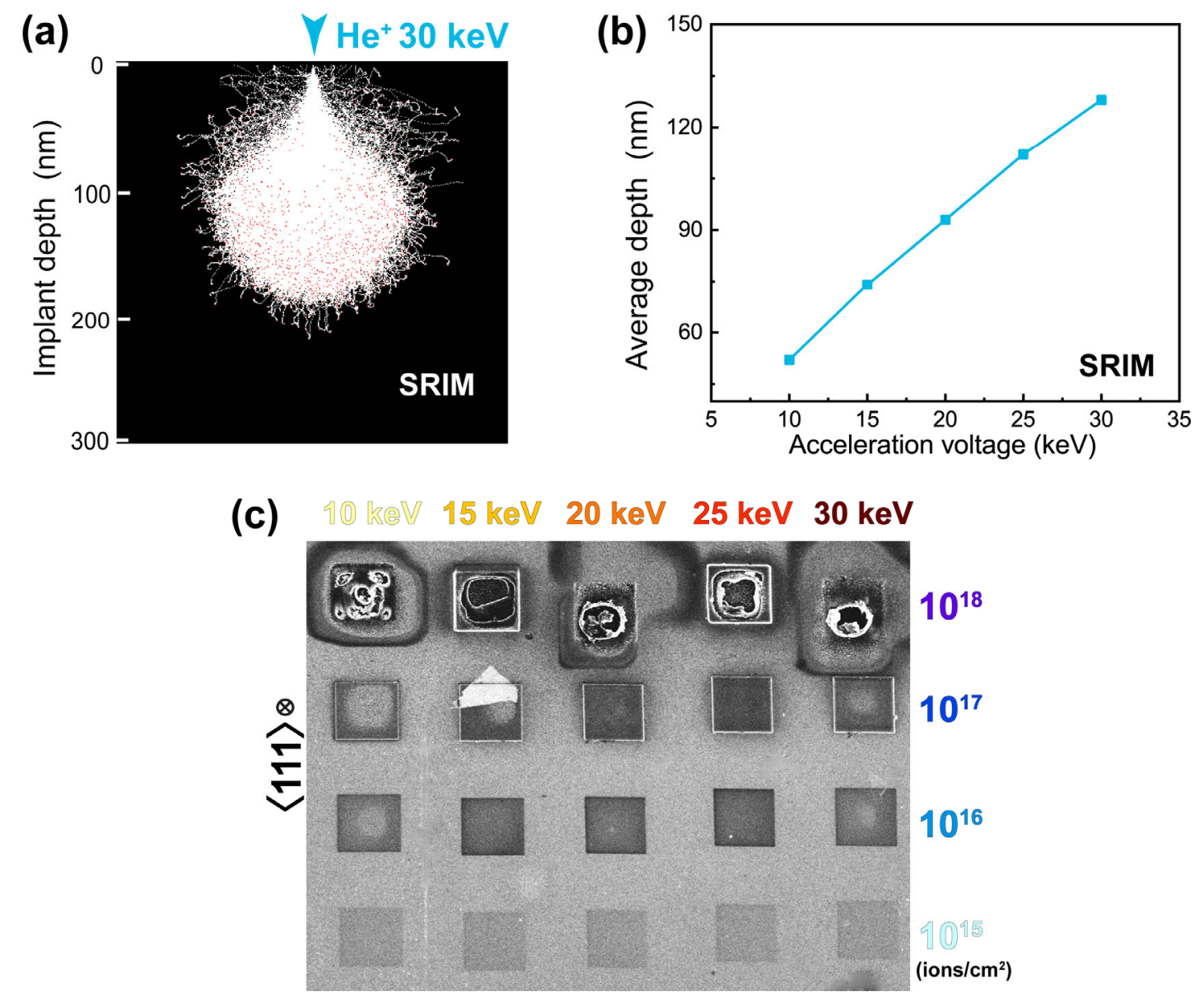

Figure 1. Characterization of $\mathrm{He}$ ion irradiation on diamond surface. Monte Carlo simulation of the distribution of 100,000 trajectories of He ions accelerated with $30 \mathrm{keV}$ in a diamond substrate (a) and the average penetration depth of $\mathrm{He}$ ion as a function of acceleration voltage (b) showing the increase of penetration depth with acceleration voltage. Secondary electron (SE) image of the irradiation array subject to various fluences and acceleration voltages on $\langle 111\rangle$-oriented single-crystal (SC) diamond in the helium ion microscopy (HIM) (c).

SEM observation shows that the exposure of the diamond to He ions with varying fluence and acceleration voltage led to a remarkable modification of the diamond surface morphology rather than material sputtering and removal. A swelling of $\langle 111\rangle\langle 111\rangle$-presenting diamond surface $(\langle 111\rangle\langle 111\rangle$ normal to the diamond surface) was observed through SEM, as shown in Fig. 1c. Surface swelling was found to be much more dependent on ion fluence than on ion energy, i.e., the acceleration voltage. Surface swelling was observable from $10^{16} \mathrm{ions} / \mathrm{cm}^{2}$ from 
different contrast in SE micrograph, and then the surface tended to rupture as the ion fluence increased to $10^{18}$ ions $/ \mathrm{cm}^{2}$, as rationalized from the craters observed after exposure (Fig. 1c).

To investigate the influence of crystalline orientation on the He ion irradiation damage, confocal Raman spectra were measured for the representing orientations of SC diamond at a range of fluence under various ion beam acceleration voltages as shown in Fig. 2a. It is readily seen from the spectra that the damage to diamond structure occurred in all orientations upon He ions irradiation, coinciding with the appearance of $\mathrm{G}$ band peak $\left(1580 \mathrm{~cm}^{-1}\right)$ of $\mathrm{sp}^{2}$ bonds and the decrease of diamond peak intensity $\left(1332 \mathrm{~cm}^{-1}\right)$ of $\mathrm{sp}^{3}$ bonds $[30,31]$. The energetic He ions break carbon-carbon bonds and also implant vacancies due to the knock-on effect on the lattice atoms. The surface swelling of diamond is attributed to the volume expansion from a dense diamond structure with $\mathrm{sp}^{3}$ bonds (density of $3.514 \mathrm{~g} \cdot \mathrm{cm}^{-3}$ ) to an expanded, damaged structure with a density in the range of $2.16-2.95 \mathrm{~g} \cdot \mathrm{cm}^{-3}$, depending on the fraction of $\mathrm{sp}^{2}$ bonds $[12,32]$. This yields a volume expansion is in the range of $19.1-62.7 \%$ for the damaged layer. With increasing He ion fluence, He bubbles formed by the agglomeration of vacancies are expected to induce further volume expansion, as observed in He ion irradiated Silicon [33]. Moreover, the $\mathrm{G}$ band peak is broadened and downshifted to $\sim 1500 \mathrm{~cm}^{-1}$ (dotted lines) at an ion fluence of $10^{17}$ ions $/ \mathrm{cm}^{2}$, indicating gradual diamond amorphization and further defect implantation [31]. This was also confirmed by the increase of full width at half maximum (FWHM) of the diamond peak with He ion fluence in all orientations - Fig. 2b. Nevertheless, no lateral shift of the diamond peak from the local strain was observed for all radiation conditions with sub-MeV acceleration voltages. This is in contrast to the downshifting observed in the diamond peak under $\mathrm{MeV}$ ion irradiation caused by tensile strains from the high concentration of defects $[34,35]$. Therefore, the strain effect of the sub-MeV ion irradiation on the diamond structure was negligible. 

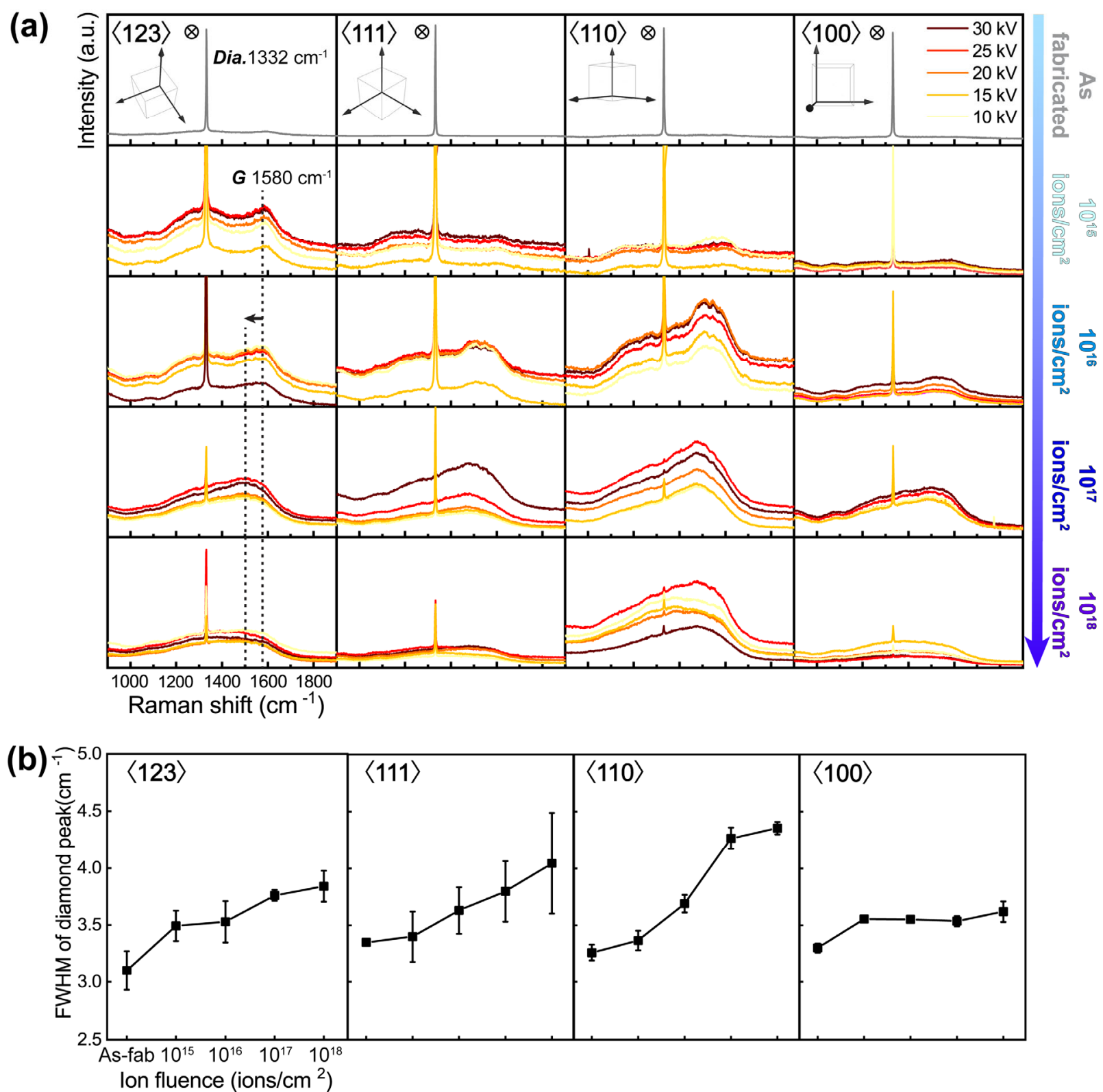

Figure 2. Raman spectroscopy of irradiated diamonds with low angle crystalline orientations. (a) Untreated confocal Raman spectra ( $633 \mathrm{~nm}$ HeNe excitation source) for each orientation of SC diamond exposed to $\mathrm{He}$ ion radiation over a range of fluences and acceleration voltages. A transition from crystalline $\mathrm{sp}^{3}$ diamond to amorphous carbon is seen with increasing ion fluence. (b) Full width at half maximum (FWHM) of diamond peak $\left(1332 \mathrm{~cm}^{-1}\right)$ as a function of $\mathrm{He}$ ion fluence.

However, the influence of He ion irradiation on the Raman spectra was observed to be dependent on the crystalline orientation. In the $\langle 123\rangle$-oriented sample, the $\mathrm{G}$ band showed a significantly higher intensity compared to other orientations at the lowest ion fluence of $10^{15}$ 
ions $/ \mathrm{cm}^{2}$. With gradually increasing ion fluence, the increase of $\mathrm{G}$ band and the decrease of diamond peak intensity indicated the amorphization of diamond as well as implantation of vacancies. Of further note, the $\mathrm{G}$ band broadening with increasing ion fluence also indicates a

high density of implanted defects after ion radiation [22]. At the highest fluence of $10^{18}$ ions $/ \mathrm{cm}^{2}$, the diamond peak was almost dissipated in $\langle 110\rangle$ and $\langle 100\rangle$ orientations at the same fluence. Nevertheless, a reemergence of the diamond peak, together with a significant reduction of the $\mathrm{G}$ band in the Raman spectra of both $\langle 123\rangle$ and $\langle 111\rangle$ orientations is observed (Fig. 2a). Consistent with the observed surface swelling and He bubbles formation during irradiation in the $\langle 111\rangle$ orientation (Fig. 1), this suggests the damaged layer at surface was at least partially sputtered away during the He-bubble formation at the mean penetration depth level of the ions for the accelerating voltage. The undamaged diamond under this gas phase layer was detected again for both $\langle 123\rangle$ - and $\langle 111\rangle$-oriented samples. In contrast, the $\langle 110\rangle$ - and $\langle 100\rangle$-oriented diamonds were gradually damaged to amorphous carbon at sufficient ion fluence. Additionally, it was also observed from the spectra in Fig. 2a that the sub-MeV acceleration voltage had negligible observable influence on the irradiation damage of the diamond structure, irrespective of the crystal orientation. According to the aforementioned SRIM simulation, the average penetration depth of He ions under various acceleration voltages is still within the range of Raman scattering depth in diamond.

\subsection{Strength degradation from He ion irradiation}

Since the Raman spectral analysis of Fig. 2a has shown that the irradiation damage on SC diamond is particularly orientation-dependent, both a damage-susceptible orientation - $\langle 123\rangle$ and a damage-tolerant orientation - $\langle 100\rangle$ - of SC diamond were chosen for nano-mechanical testing to study the effects of ion irradiation on the resulting mechanical properties. The morphologies of $\langle 123\rangle$ - and $\langle 100\rangle$-oriented pillars before and after ion radiation are 
respectively shown in Fig. 3a and 3b. This top-down ion implantation was implemented by placing a square pattern with size of $1 \times 1 \mu \mathrm{m}^{2}$ above the apex of cylindrical pillar (diameter of $0.5 \mu \mathrm{m})$. Actually, the side surface of pillar was also exposed to He ions during top-down radiation due to the taper of pillar (angle of $\sim 4^{\circ}$ ) labelled in Fig. 3a, and then slightly swelled up along the radial direction of pillar. For pillars from each orientation in Fig. 3, the secondary electron micrographs were taken from different pillars with various ion fluences. Although the same parameters, i.e. the beam voltage, current and pattern size, were identically applied to all pillars during FIB milling, the base of each pillar was still different due to the manual overlap of milling patterns after each milling current and also the subsequent helium ion irradiation to different fluences in the HIM. However, the pillar geometries were consistent after FIB milling according to post-mortem measurements. Therefore, the influence of the variation in base geometry on microcompression behavior is taken to be negligible, since the fracture of diamond pillars occurred mainly in the irradiated, upper part of pillars according to the observation after compression shown in Fig. 4c. After ion irradiation, swelling was predominately observed in the upper portion of diamond pillars. The swelling degree was found to be larger in $\langle 123\rangle$-oriented pillars compared to pillars with $\langle 100\rangle$ orientation. For the $\langle 123\rangle$ oriented pillars, the average diameter is $702.8 \pm 19.1 \mathrm{~nm}$, i.e., $\sim 50 \%$ increase in diameter compared to the as-fabricated pillars. For the $\langle 100\rangle$-oriented pillars, the average diameter is $596.3 \pm 14.9 \mathrm{~nm}$, i.e., $\sim 20 \%$ expansion in diameter. This was calculated by averaging the diameter at the top, middle and base of each irradiated pillars from both orientations. 


\section{As-fabricated $\quad 10^{16}$ ions $/ \mathrm{cm}^{2} \quad 10^{17}$ ions $/ \mathrm{cm}^{2} \quad 10^{18}$ ions $/ \mathrm{cm}^{2}$}

(a)
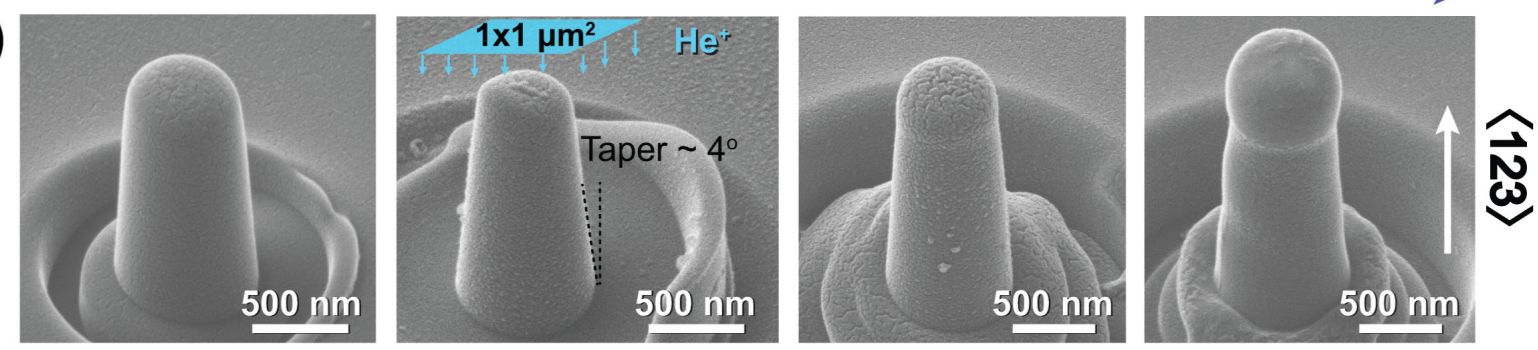

(b)
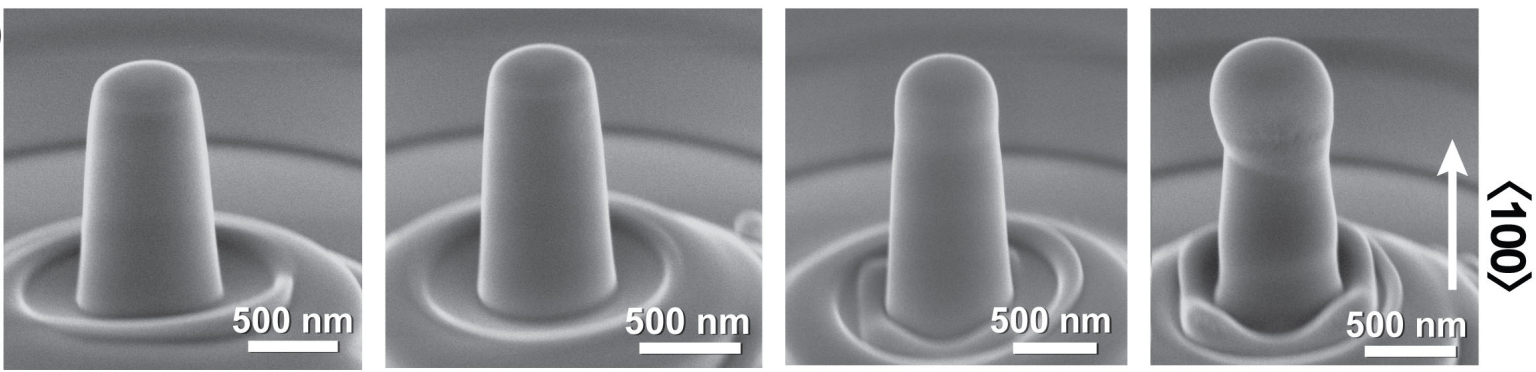

Figure 3. FIB-machined pillars with orientations of $\langle 123\rangle$ (a) and $\langle 100\rangle$ (b) irradiated to different fluences using $30 \mathrm{keV}$ acceleration voltage and a square pattern of $1 \times 1 \mu \mathrm{m}^{2}$.

As negligible plasticity was observed during compression. The pillar geometry remained approximately constant with negligible elastically transverse expansion, expressed in the small Poisson's ratio of diamond of 0.07 [36]. Thus, the stress-strain curves calculated using the top diameter of pillar represented the highest stress experienced by the pillars during loading (solid line, Fig. 4). However, the pillars irradiated with the highest fluence of $10^{18}$ ions $/ \mathrm{cm}^{2}$ show their smallest diameter just below the swollen upper part of the pillar due to the volume expansion (Fig. 3), which is about of $528 \pm 3 \mathrm{~nm}$ for $\langle 100\rangle$-oriented pillars and $585 \pm 19 \mathrm{~nm}$ for $\langle 123\rangle$-oriented pillars. So the stress-strain curves of pillars with the highest fluence of $10^{18}$ ions $/ \mathrm{cm}^{2}$ were also calculated with the smallest diameter in the upper part of the pillars for comparison (dashed line, Fig. 4). The upper region of the pillars was also the region which experienced the most concentrated fluence of radiation, as determined by SRIM. As shown in the flow curves from two orientations in Fig. $\mathbf{4 a}$ and $\mathbf{4 b}$, both as-fabricated and irradiated pillars were extremely brittle as catastrophic failure after the linear elastic regime during compression. 
In as-fabricated pillars, the measured Young's modulus $(E)$ of $\langle 123\rangle$-oriented pillar $(\sim 1400$ GPa) was higher than theoretical modulus $(\sim 1204 \mathrm{GPa})[37,38]$. This is attributed to a combination of the tapered pillar geometry and the pressure dependence of the modulus of diamond at stresses close to $200 \mathrm{GPa}[21,37,39]$. At these high stresses, the covalent bonds can be strengthened beyond their normal configuration [40]. However, the measured $E$ of $\langle 100\rangle$-oriented pillar $(\sim 1048 \mathrm{GPa})$ was approximately consistent with the theoretical value $(\sim 1052 \mathrm{GPa})[41]$. This is probably due to the lower stress $(<100 \mathrm{GPa})$ sustained by the $\langle 100\rangle$ oriented pillars during compression before failure.

Figure 4. Deformation behavior of as-fabricated and irradiated SC diamond pillars. Engineering stressstrain curves of $\langle 123\rangle$-oriented (a) and $\langle 100\rangle$-oriented (b) pillars. Solid lines were calculated with the top diameter of as-fabricated pillar, while the dashed lines were calculated with the narrowest diameter below the swollen upper region of the pillars with the highest fluence of $10^{18}$ ions $/ \mathrm{cm}^{2}$. Secondary electron micrographs of failure morphologies of $\langle 100\rangle$-oriented pillars irradiated with various ion
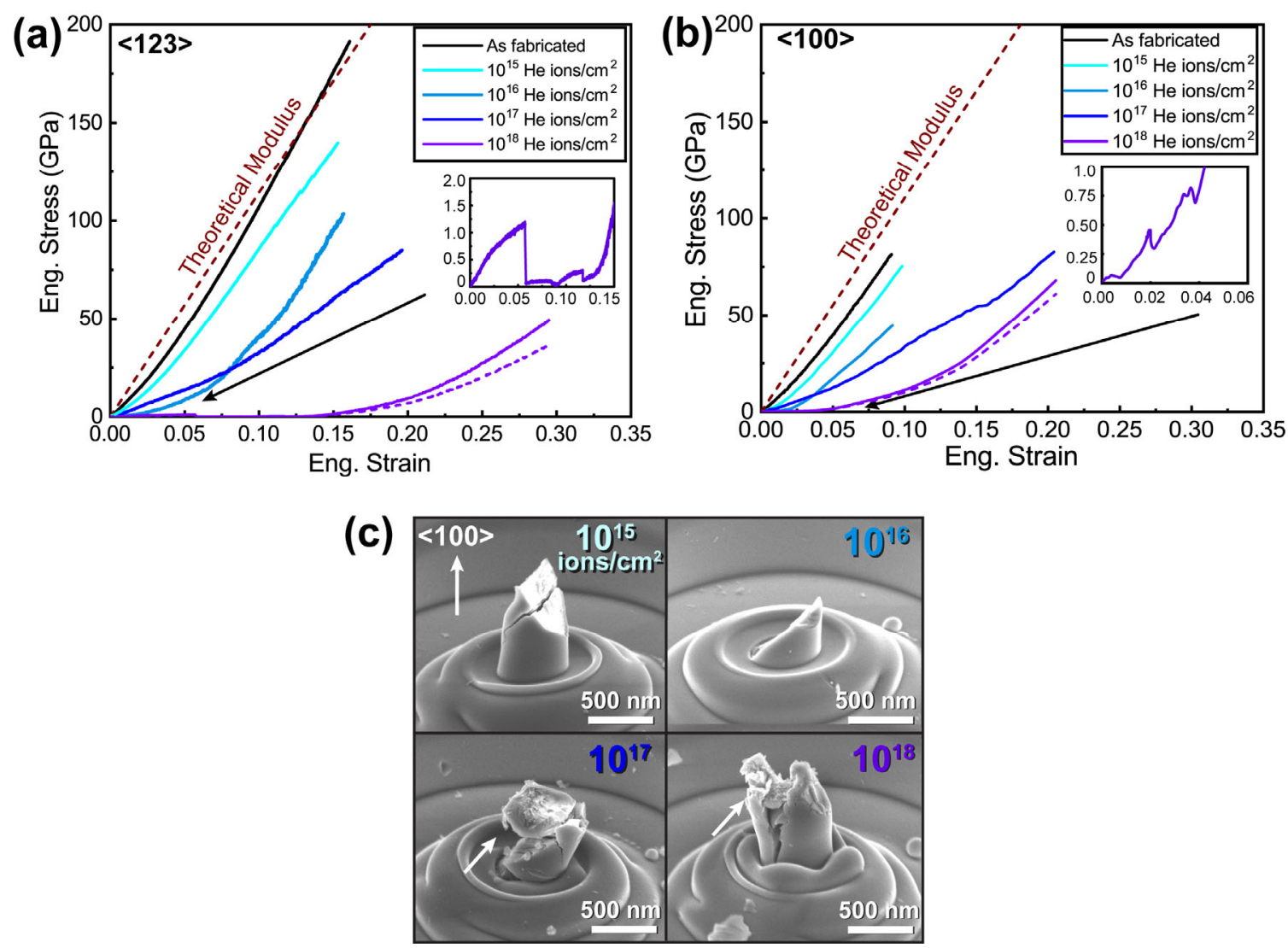

fluences (c). 
In the ion-irradiated pillars, the relative Young's modulus is observed to progressively decrease with increasing He ion fluence (hollow symbol, Fig. 5a). This results from irradiation damage to the crystal lattice, breaking stronger $\mathrm{sp}^{3}$ bonds into $\mathrm{sp}^{2}$ bonds with lower stiffness, i.e., amorphous carbon ( $E \sim 40 \mathrm{GPa})[42,43]$. Additionally, He bubbles were also extensively implanted into diamond, resulting in a porous structure with initially nano-sized bubbles which then coalesce to form larger bubbles with increasing ion fluence (Fig. 3a). Normally, the porosity in target materials after ion irradiation has a logarithmic dependency on the ion fluence, i.e., $\sim \ln ($ fluence $)[44,45]$. The fitting relationship between the relative Young's modulus $\left(E / E_{o}\right)$ and He ion fluence displays good agreement to the porosity model (dash curves, Fig. 5a) in ceramics, i.e., $E / E_{O} \sim 1-a \cdot \ln ($ fluence $+b)$ where $E_{o}$ is the Young's modulus of asfabricated SC diamond, $a$ and $b$ are the constants, for both $\langle 123\rangle$ and $\langle 100\rangle$ orientations [46]. The flow curves of irradiated pillars at the higher ion fluence of $10^{17}$ and $10^{18}$ ions $/ \mathrm{cm}^{2}$ notably deviated from linear behavior at the large strain. Because the irradiation damages were concentrated at the first hundreds of nanometers at the top part of irradiated pillars. There were several stress drop due to the bubbles fracture after 5\% strain (inset, Fig. 4a). So the Young's moduli were only calculated until 5\% of engineering strain in these pillars with higher fluences $\left(10^{17}\right.$ and $10^{18}$ ions $\left./ \mathrm{cm}^{2}\right)$ for both orientations.

The failure behavior of irradiated pillars is also observed to be orientation-dependent. In the $\langle 123\rangle$-oriented pillars, the initial portion of the stress-strain curves for the pillars irradiated at the highest fluence of $10^{18}$ ions $/ \mathrm{cm}^{2}$ shows a very low stiffness. This is attributed to the compression of a large He bubble at the top of the sample (Supplementary Video 1). The pillar was observed in situ to fracture with a sharp stress drop (Fig. 4a inset) due to the displacement control of the system. The indenter then regained contact with the underlying diamond pillar after the remnants of the amorphous carbon bubble are compressed away. This acts to offset the stress-strain curve along the strain axis. Under this amorphized bubble, the 
irradiated pillar is revealed in situ to have a geometry similar to the as-fabricated pillars. This suggests the stress levels experienced in the may be closer to those calculated using the original top diameter, rather than the smallest diameter below the swollen part of the pillar. After bubble fracture, the stiffness was increased a bit but still smaller than the as-fabricated pillars and the irradiated pillars with lower fluences (Fig. 4a). Because the underlying pillar was also partially damaged during irradiation. But the damage level of lower part was less intensive than the top part. Moreover, the debris from the fracture of the upper pillar were flattened under the flat punch during the compression.

Similar behavior is seen in the compression of $\langle 100\rangle$-oriented pillars (Supplementary Video 2), but the stress drop is smaller (Fig. 4b inset) due to smaller He bubbles, as shown in Fig. 3a and 3b. In addition, the decrease in the relative Young's modulus with increasing ion fluence is less pronounced in the $\langle 100\rangle$-oriented pillars (Fig. 5a). The failure behavior of the pillars irradiated at lower fluences, i.e., $10^{15}$ and $10^{16}$ ions $/ \mathrm{cm}^{2}$, was similar to as-fabricated pillars, showing fracture with sharp $\{111\}$ cleavage planes (Fig. 4c) [36]. However, pillars irradiated to higher fluences, i.e., $10^{17}$ and $10^{18}$ ions $/ \mathrm{cm}^{2}$, showed greater fragmentation of the pillar after compression (arrows, Fig. 4c). This is attributed to comminution of the heavily damaged upper region of the pillar during failure. Critically, the influence of ion irradiation on the failure strength of SC diamond is remarkably orientation-dependent shown in Fig. $\mathbf{5 b}$. In $\langle 123\rangle$-oriented pillars, the failure stress of microcompression until fracture, $\sigma$, decreases following an exponential trend with increasing ion fluence. In contrast, the failure stress in $\langle 100\rangle$-oriented pillars only slightly decreases with increasing ion fluence. At the highest fluence level of $10^{18}$ ions $/ \mathrm{cm}^{2}$, the failure stress of the $\langle 123\rangle$-oriented pillars reduced to only $27 \%$ of its as-fabricated strength, whereas the $\langle 100\rangle$ orientation retained $72 \%$ of its strength. Since the volume expansion was notable at the upper part of pillars with the highest fluence of $10^{18}$ ions $/ \mathrm{cm}^{2}$, the diameter at the narrowest part of the pillars was also used to calculate the failure 
stress (hollow symbols, Fig. 5b). This induced a small decrease of the failure stress, but the values are still consistent with the observed trends with fluence.
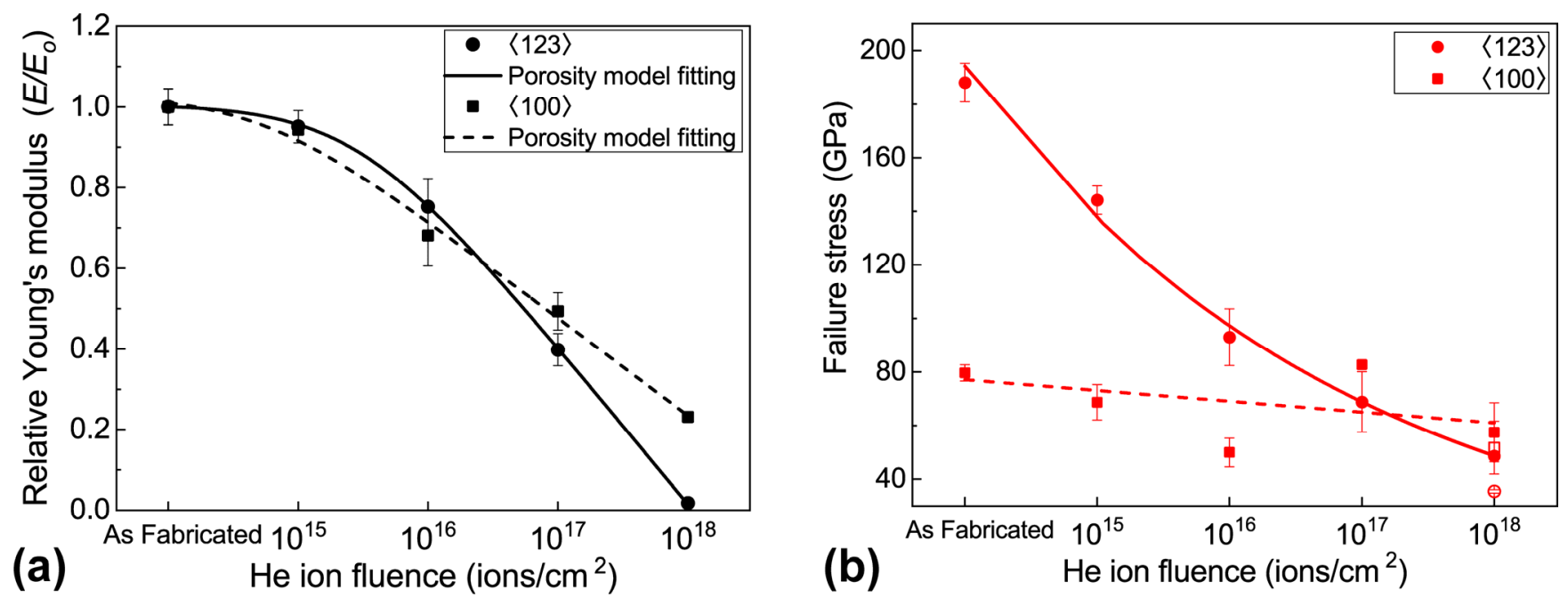

Figure 5. Micromechanical properties of as-fabricated and irradiated diamond pillars. Relative Young's modulus (a) and failure stress (b) of diamond pillars as a function of He ion fluence. The solid symbols of failure stress were calculated with the top diameter of pillars, while the hollow symbols of failure stress were calculated with the narrowest diameter of the pillars irradiated at the highest fluence of $10^{18}$ ions $/ \mathrm{cm}^{2}$ for both orientations.

The mechanical properties of irradiated SC diamonds were shown to be highly orientation-dependent. This difference in irradiation damage is attributed to the effect of crystal orientation on ion channeling, which allows energetic ions to travel greater distances into the crystalline lattice along open channels with minimal interaction with lattice atoms [47]. In the absence of such channels, energetic ions directly collide with atoms in the diamond lattice, altering $\mathrm{sp}^{3}$ bonds into $\mathrm{sp}^{2}$ bonds and implanting points defects, such as vacancies, reducing crystallinity and increasing disorder in the diamond lattice $[32,48]$. Ion channeling can only occur if crystal is oriented within a critical angle, $\psi$, of the direction of incident ions from the perfect channeling axis, as proposed by Lindhard et al. [49]. In SC diamond, both axial and planar channeling collectively contributed to the reducing the influence of radiation damage.[50] The axial channeling occurs in the low-index crystalline orientations with high symmetry, whereas the planar channeling emerges in the orientations between the low-index 
ones [51]. The critical angles were evaluated according to Monte Carlo calculation by Barrett et al. [52]:

$\psi_{\text {axial }}=\left(\frac{3 \cdot a^{2} \cdot z_{1} \cdot z_{2} \cdot e^{2}}{E \cdot d^{3}}\right)^{1 / 4}$

$(2)$,

$\psi_{\text {planar }}=0.93 \cdot\left(\frac{2 \pi \cdot Z_{1} \cdot Z_{2} \cdot e^{2} \cdot a \cdot N \cdot d_{p}}{E}\right)^{1 / 2}$

where $Z_{1}$ and $Z_{2}$ are the atomic numbers of ions and the target atoms, respectively, $e^{2}$ is the square of the elementary charge and equal to $14.4 \mathrm{eV} \cdot \mathrm{m}^{-10}$ [47], $E$ is the acceleration voltage of ions $(30 \mathrm{keV}), d$ is the distance between two atoms along the channel wall, $d_{p}$ is the planar spacing between channel planes, and $N$ is the number of carbon atoms per unit volume, $a=$ $a_{B} \cdot 0.8853 \cdot\left(Z_{1}{ }^{2 / 3}+Z_{2}{ }^{2 / 3}\right)^{1 / 4}$, where $a_{B}$ is the Bohr radius. The calculated critical angles are listed in Tab. 1. The results show the $\psi_{\text {axial }}$ in $\langle 123\rangle$ orientation is only half the value of the other low-index orientations, indicating lower channeling efficiency of $\mathrm{He}$ ions in this orientation. Although the value of $\psi_{\text {planar }}$ is approximately similar $\left(\sim 2.3^{\circ}\right)$ in all investigated orientations, the critical angle $\psi$ is overall smallest in the $\langle 123\rangle$ orientation. This finding is consistent with results of proton channeling in SC diamond, where the ratio of backscattered protons was found to be significantly greater in the $\langle 123\rangle$ orientation due to low channeling efficiency [50, 53].

Table 1. Critical angles of axial and planar channelings in single crystal diamond with representing orientations.

\begin{tabular}{lcccc}
\hline & $\langle 110\rangle$ & $\langle 100\rangle$ & $\langle 111\rangle$ & $\langle 123\rangle$ \\
\hline$\psi_{\text {axial }}(\mathrm{deg})$ & 4.7 & 4.0 & 4.0 & 2.0 \\
\hline$\psi_{\text {planar }}(\mathrm{deg})$ & 2.3 & 2.2 & 2.3 & 2.3 \\
\hline
\end{tabular}


Regarding the profile of focused He ion beam, the convergence angle is $\sim 1$ degree, i.e., smaller than all calculated ciritical angles for ion channeling in the representing orientations [54]. Thus, the majority of incident He ions are able to channel into the diamond lattice in the four tested orientations. This channeling behavior can be more easily apprehended by considering the head-on view of the lattice orientations shown in Figure 6a-d. The $\langle 110\rangle,\langle 111\rangle$ and $\langle 100\rangle$ orientations present larger channels (orange arrows) for incident ions [55], whereas the channels in the $\langle 123\rangle$ orientation are extremely narrow. This offers an explanation for the greater He ion irradiation damage in $\langle 123\rangle$-oriented diamond, which is seen both in terms of the reduction of strength (Figure 5b) and in the observation of $\mathrm{G}$ bands at quite low fluence of $10^{15}$ ions $/ \mathrm{cm}^{2}$ in the Raman spectra (Fig. 2a). In contrast, the $\langle 100\rangle$ orientation, with higher channeling efficiency, exhibited better ion radiation resistance through lower strength degradation and less structural damage apparent in the Raman spectra. 

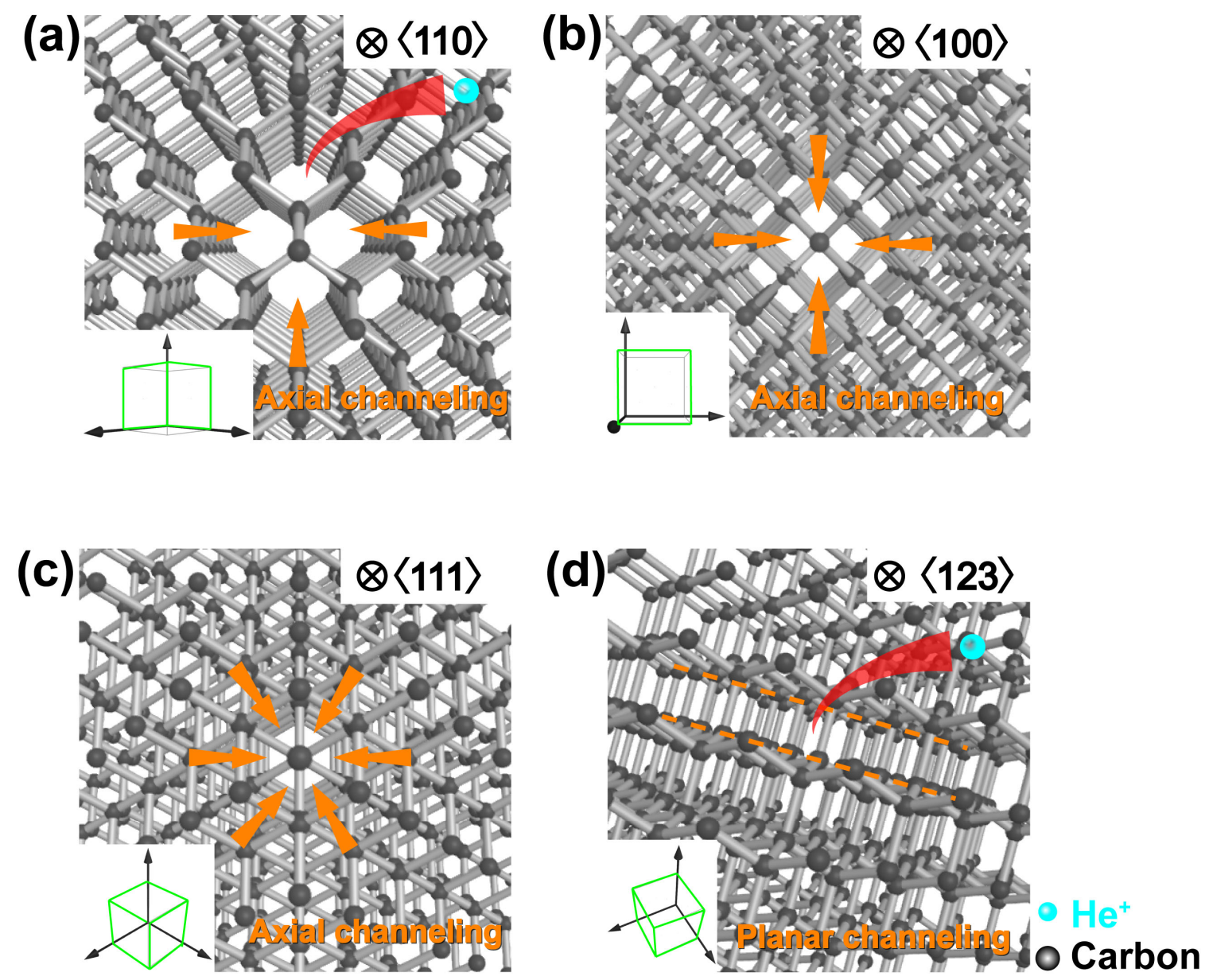

Figure 6. Ion channeling in various orientations of the diamond lattice. Head-on view of open channels for incident ions in SC diamond lattice with representing orientations of (a) $\langle 110\rangle$, (b) $\langle 100\rangle$, (c) $\langle 111\rangle$ and (d) $\langle 123\rangle$ out of plane.

However, the influence of the crystalline orientation on He ion irradiation damage is conventionally neglected for materials with anisotropic crystal structures. Previously, ion irradiation was only considered to isotropically amorphize crystals and implant nanobubbles. In aforementioned Monte Carlo simulation (SRIM), the average penetration depth of He ions in diamond is determined to be $\sim 128 \mathrm{~nm}$ with an acceleration voltage of $30 \mathrm{keV}$. This simulation result is based on the assumption that target materials are both continuous and isotropic to the incident ions and neglects the anisotropy of the crystal lattice $[26,56]$. However, the actual penetration depth of He ions within the critical angle $\psi$ is significantly deeper because of the ion channeling effect [47]. For non-channeling ions, the penetration of the energetic He ions would be stopped at the shallow depth determined by SRIM simulation due 
to collisions with the diamond lattice. In more isotropic FCC metals, like copper, micromechanical tests after $\mathrm{He}$ ion irradiation showed that the strength and ductility were increased due to the interaction between dislocations and He bubbles [57, 58]. For SC silicon with the same diamond-cubic structure, the strength was decreased because of defect-driven amorphization after exposure to a fluence over the threshold value $\left(\sim 10^{17}\right.$ ions $\left./ \mathrm{cm}^{2}\right)$, but ductility was improved by shear band in the amorphous phase $[33,59]$. In this work, where orientation is considered, the ion radiation damage on the strength of diamond is shown to be significantly orientation-dependent (Fig. 5b). In the $\langle 123\rangle$ orientation, the diamond structure ( $\mathrm{sp}^{3}$ bonds) is more effectively transformed into amorphous carbon with $\mathrm{sp}^{2}$ bonds at lower fluences due to a lower amount of ion channeling in comparison with the other orientations [60].

In previous studies, this transition is dependent on the level of implanted point defects as well as tensile strain from implanted point defects from ion irradiation, by which $\mathrm{sp}^{3}$ bonds are collapsed into $\mathrm{sp}^{2}$ bonds as the stress exceeded the tensile strength of the diamond lattice [32]. Actually, this stress due to the irradiation induced tensile strain is higher in $\langle 123\rangle$ orientation because of the higher elastic modulus of this orientation (15\% higher than $\langle 100\rangle$ orientation). Therefore, irradiation damage is more pronounced in $\langle 123\rangle$-oriented SC diamond due to both less ion channeling as well as higher tensile stress to damage the diamond structure. After the transition of covalent bonds, He bubbles are successively generated by clustering and coalescence of vacancies and He point defects with increasing ion fluence to further degrade the strength of diamond structure. 


\section{Conclusion}

In this study, the effect of crystalline orientation on $\mathrm{He}$ ion irradiation damage was systematically investigated using Raman spectroscopy and micromechanical testing. Radiation damage was implemented by employing a helium ion microscope (HIM) with a keV source to expose SC diamond surfaces with typical low angle crystallographic orientations to a focused He ion beam at varying fluence and acceleration voltage. The observed surface swelling after ion irradiation was due to the volume expansion in the damage layer and He bubbles formation. The degree of swelling was observed to be more dependent on the ion fluence rather than acceleration voltage. Raman spectra demonstrated the $\langle 123\rangle$-oriented SC diamond was more sensitive to radiation as the transition of $\mathrm{sp}^{3}$ to $\mathrm{sp}^{2}$ bonds at low fluence compared to other lowindex orientations. The subsequent micromechanical testing indicated the strength of $\langle 123\rangle$ oriented diamond was exponentially decreased with increasing the ion fluence, while the $\langle 100\rangle$ oriented diamond showed greater resistance to ion radiation. Hence, structural variation and strength degradation due to irradiation damage in diamond is highly orientation-dependent. This is rationalized by the level of irradiation damage to diamond structure and resulting mechanical properties being significantly dependent on the ion channeling efficiency as well as the elastic modulus of corresponding crystalline orientation. This study offers the essential knowledge for precise surface patterning of diamond with different orientations for nanofabrication.

\section{Acknowledgement}


The authors are grateful for the financial support of the Swiss National Science Foundation (SNF Grant: 200021_166094). I.S. is thankful to Empa for financial support and to Swiss National Science Foundation for support in equipment procurement (REquip 206021_133823). The authors would like to thank P. Gasser and K. Kunze (ScopeM, ETH Zurich) for their help in the sample preparation using FIB and HRSEM characterization.

\section{Notes}

The authors declare no competing financial interest. 


\section{References}

[1] A.V. Inyushkin, A.N. Taldenkov, V.G. Ralchenko, A.P. Bolshakov, A.V. Koliadin, A.N. Katrusha, Thermal conductivity of high purity synthetic single crystal diamonds, Physical Review B 97(14) (2018).

[2] J.E. Field, The properties of natural and synthetic diamond, Academic Press 1992.

[3] P. Neumann, I. Jakobi, F. Dolde, C. Burk, R. Reuter, G. Waldherr, J. Honert, T. Wolf, A. Brunner, J.H. Shim, High-precision nanoscale temperature sensing using single defects in diamond, Nano letters 13(6) (2013) 2738-2742.

[4] M.A. Davies, C.J. Evans, R.R. Vohra, B.C. Bergner, S.R. Patterson, Application of precision diamond machining to the manufacture of microphotonics components, Lithographic and micromachining techniques for optical component fabrication II, International Society for Optics and Photonics, 2003, pp. 94-109.

[5] M. Héritier, A. Eichler, Y. Pan, U. Grob, I. Shorubalko, M.D. Krass, Y. Tao, C.L. Degen, Nanoladder cantilevers made from diamond and silicon, Nano letters 18(3) (2018) 18141818.

[6] R.W. Tustison, J.M. Dodson, J.R. Brandon, H.K. Dhillon, I. Friel, S.L. Geoghegan, T.P. Mollart, P. Santini, G.A. Scarsbrook, D.J. Twitchen, A.J. Whitehead, J.J. Wilman, H. de Wit, Single crystal and polycrystalline CVD diamond for demanding optical applications, 8016 (2011) 80160L.

[7] P. Olivero, S. Rubanov, P. Reichart, B.C. Gibson, S.T. Huntington, J. Rabeau, A.D. Greentree, J. Salzman, D. Moore, D.N. Jamieson, S. Prawer, Ion-Beam-Assisted Lift-Off Technique for Three-Dimensional Micromachining of Freestanding Single-Crystal Diamond, Advanced Materials 17(20) (2005) 2427-2430.

[8] M. Draganski, E. Finkman, B. Gibson, B. Fairchild, K. Ganesan, N. Nabatova-Gabain, S. Tomljenovic-Hanic, A. Greentree, S. Prawer, Tailoring the optical constants of diamond by ion implantation, Optical Materials Express 2(5) (2012) 644-649.

[9] L. Sekaric, J. Parpia, H. Craighead, T. Feygelson, B. Houston, J. Butler, Nanomechanical resonant structures in nanocrystalline diamond, Applied Physics Letters 81(23) (2002) 44554457.

[10] J.W. Mayer, Ion implantation in semiconductors, 1973 International Electron Devices Meeting, IEEE, 1973, pp. 3-5.

[11] O.N. Lopatin, A.G. Nikolaev, V.F. Valeev, V.I. Nuzhdin, R.I. Khaibullin, CrystalChemical Features of Diamonds Implanted with Helium Ions, Crystallography Reports 63(3) (2018) 327-330.

[12] M. Piccardo, F. Bosia, P. Olivero, N. Pugno, An analytical model for the mechanical deformation of locally graphitized diamond, Diamond and Related Materials 48 (2014) 7381.

[13] J.D. Hunn, C.P. Christensen, Ion beam and laser-assisted micromachining of singlecrystal diamond, Solid State Technology 37(12) (1994) 57-61.

[14] E. Bloom, S. Zinkle, F. Wiffen, Materials to deliver the promise of fusion powerprogress and challenges, Journal of nuclear materials 329 (2004) 12-19.

[15] A. Kasugai, K. Sakamoto, K. Takahashi, M. Tsuneoka, T. Kariya, T. Imai, O. Braz, M. Thumm, J.R. Brandon, R.S. Sussman, A. Beale, D.C. Ballington, Chemical vapor deposition diamond window for high-power and long pulse millimeter wave transmission, Review of Scientific Instruments 69(5) (1998) 2160-2165.

[16] D. Kania, M. Landstrass, M. Plano, L. Pan, S. Han, Diamond radiation detectors, Diamond and Related Materials 2(5-7) (1993) 1012-1019.

[17] M.S. Joens, C. Huynh, J.M. Kasuboski, D. Ferranti, Y.J. Sigal, F. Zeitvogel, M. Obst, C.J. Burkhardt, K.P. Curran, S.H. Chalasani, L.A. Stern, B. Goetze, J.A. Fitzpatrick, Helium 
Ion Microscopy (HIM) for the imaging of biological samples at sub-nanometer resolution, Sci Rep 3 (2013) 3514.

[18] B.W. Ward, J.A. Notte, N.P. Economou, Helium ion microscope: A new tool for nanoscale microscopy and metrology, Journal of Vacuum Science \& Technology B: Microelectronics and Nanometer Structures 24(6) (2006) 2871.

[19] I. Shorubalko, L. Pillatsch, I. Utke, Direct-Write Milling and Deposition with Noble Gases, Helium Ion Microscopy, Springer2016, pp. 355-393.

[20] U. Gerlach-Meyer, J. Coburn, E. Kay, Ion-enhanced gas-surface chemistry: The influence of the mass of the incident ion, Surface Science 103(1) (1981) 177-188.

[21] J.M. Wheeler, R. Raghavan, J. Wehrs, Y. Zhang, R. Erni, J. Michler, Approaching the Limits of Strength: Measuring the Uniaxial Compressive Strength of Diamond at Small Scales, Nano Lett 16(1) (2016) 812-6.

[22] T. Dieing, O. Hollricher, J. Toporski, Confocal raman microscopy, Springer2011. [23] W. Kang, M. Merrill, J.M. Wheeler, In situ thermomechanical testing methods for micro/nano-scale materials, Nanoscale 9(8) (2017) 2666-2688.

[24] J. Wheeler, J. Michler, Elevated temperature, nano-mechanical testing in situ in the scanning electron microscope, Review of Scientific Instruments 84(4) (2013) 045103.

[25] M. Chen, J. Wehrs, J. Michler, J.M. Wheeler, High-Temperature In situ Deformation of GaAs Micro-pillars: Lithography Versus FIB Machining, Jom 68(11) (2016) 2761-2767.

[26] J.F. Ziegler, M.D. Ziegler, J.P. Biersack, SRIM-The stopping and range of ions in matter (2010), Nuclear Instruments and Methods in Physics Research Section B: Beam Interactions with Materials and Atoms 268(11) (2010) 1818-1823.

[27] J.C. Bourgoin, B. Massarani, Threshold energy for atomic displacement in diamond, Physical Review B 14(8) (1976) 3690-3694.

[28] Y. Kudriavtsev, A. Villegas, A. Godines, R. Asomoza, Calculation of the surface binding energy for ion sputtered particles, Applied Surface Science 239(3-4) (2005) 273-278. [29] W.R. McKenzie, M.Z. Quadir, M.H. Gass, P.R. Munroe, Focused Ion beam implantation of diamond, Diamond and Related Materials 20(8) (2011) 1125-1128.

[30] K. Ushizawa, K. Watanabe, T. Ando, I. Sakaguchi, M. Nishitani-Gamo, Y. Sato, H. Kanda, Boron concentration dependence of Raman spectra on $\{100\}$ and $\{111\}$ facets of Bdoped CVD diamond, Diamond and Related Materials 7(11) (1998) 1719-1722.

[31] A.C. Ferrari, J. Robertson, Raman spectroscopy of amorphous, nanostructured, diamond-like carbon, and nanodiamond, Philosophical Transactions of the Royal Society of London A: Mathematical, Physical and Engineering Sciences 362(1824) (2004) 2477-2512. [32] B.A. Fairchild, S. Rubanov, D.W. Lau, M. Robinson, I. Suarez-Martinez, N. Marks, A.D. Greentree, D. McCulloch, S. Prawer, Mechanism for the amorphisation of diamond, Adv Mater 24(15) (2012) 2024-9.

[33] Y.C. Wang, L. Tian, F. Liu, Y.B. Qin, G. Zheng, J.T. Wang, E. Ma, Z.W. Shan, Helium Ion Microscope Fabrication Causing Changes in the Structure and Mechanical Behavior of Silicon Micropillars, Small (2016).

[34] K. Nugent, S. Prawer, Confocal Raman strain mapping of isolated single CVD diamond crystals, Diamond and related materials 7(2-5) (1998) 215-221.

[35] J. Orwa, K. Nugent, D. Jamieson, S. Prawer, Raman investigation of damage caused by deep ion implantation in diamond, Physical Review B 62(9) (2000) 5461.

[36] J.E. Field, The mechanical and strength properties of diamond, Rep Prog Phys 75(12) (2012) 126505.

[37] H.J. McSkimin, P. Andreatch, Elastic Moduli of Diamond as a Function of Pressure and Temperature, Journal of Applied Physics 43(7) (1972) 2944-2948.

[38] J. Rösler, H. Harders, M. Bäker, Mechanical behaviour of engineering materials: metals, ceramics, polymers, and composites, Springer Science \& Business Media2007. 
[39] F. Zhi-Jian, J. Guang-Fu, C. Xiang-Rong, G. Qing-Quan, First-principle calculations for elastic and thermodynamic properties of diamond, Communications in Theoretical Physics 51(6) (2009) 1129.

[40] F. Occelli, P. Loubeyre, R. LeToullec, Properties of diamond under hydrostatic pressures up to $140 \mathrm{GPa}$, Nat Mater 2(3) (2003) 151-4.

[41] J. Field, The mechanical and strength properties of diamond, Reports on Progress in Physics 75(12) (2012) 126505.

[42] A. Albiez, R. Schwaiger, Size Effect on the Strength and Deformation Behavior of Glassy Carbon Nanopillars, Mrs Advances 4(2) (2019) 133-138.

[43] C. Liu, Y. Lin, Z. Zhou, K.-Y. Li, Dual phase amorphous carbon ceramic achieves theoretical strength limit and large plasticity, Carbon 122 (2017) 276-280.

[44] T.T. Tran, H.S. Alkhaldi, H.H. Gandhi, D. Pastor, L.Q. Huston, J. Wong-Leung, M.J. Aziz, J.S. Williams, Suppression of ion-implantation induced porosity in germanium by a silicon dioxide capping layer, Applied Physics Letters 109(8) (2016) 082106.

[45] H.S. Alkhaldi, F. Kremer, T. Bierschenk, J.L. Hansen, A. Nylandsted-Larsen, J.S. Williams, M.C. Ridgway, Porosity as a function of stoichiometry and implantation temperature in Ge/Si1-xGex alloys, Journal of Applied Physics 119(9) (2016) 094303. [46] A. Boccaccini, Z. Fan, A new approach for the Young's modulus-porosity correlation of ceramic materials, Ceramics International 23(3) (1997) 239-245.

[47] G.S. Was, Fundamentals of radiation materials science: metals and alloys, Springer2016. [48] H. Ma, Y. Zou, A.S. Sologubenko, R. Spolenak, Copper thin films by ion beam assisted deposition: Strong texture, superior thermal stability and enhanced hardness, Acta Materialia 98 (2015) 17-28.

[49] J. Lindhard, Influence of crystal lattice on motion of energetic charged particles, Munksgaard1965.

[50] T. Derry, R. Fearick, J. Sellschop, Ion channeling in natural diamond. II. Critical angles, Physical Review B 26(1) (1982) 17.

[51] K. Nordlund, F. Djurabekova, G. Hobler, Large fraction of crystal directions leads to ion channeling, Physical Review B 94(21) (2016).

[52] J.H. Barrett, Monte Carlo channeling calculations, Physical Review B 3(5) (1971) 1527.

[53] T. Derry, R. Fearick, J. Sellschop, Ion channeling in natural diamond. Minimum yields, Physical Review B 24(7) (1981) 3675.

[54] R. Hill, J. Notte, B. Ward, The ALIS He ion source and its application to high resolution microscopy, Physics Procedia 1(1) (2008) 135-141.

[55] M.T. Robinson, O.S. Oen, Computer Studies of the Slowing Down of Energetic Atoms in Crystals, Physical Review 132(6) (1963) 2385-2398.

[56] J.F. Ziegler, J. Biersack, U. Littmark, The stopping and range of ions in solids, vol. 1, Pergamon, New York, 1985.

[57] N. Li, N.A. Mara, Y.Q. Wang, M. Nastasi, A. Misra, Compressive flow behavior of Cu thin films and $\mathrm{Cu} / \mathrm{Nb}$ multilayers containing nanometer-scale helium bubbles, Scripta Materialia 64(10) (2011) 974-977.

[58] M.S. Ding, J.P. Du, L. Wan, S. Ogata, L. Tian, E. Ma, W.Z. Han, J. Li, Z.W. Shan, Radiation-Induced Helium Nanobubbles Enhance Ductility in Submicron-Sized SingleCrystalline Copper, Nano Lett 16(7) (2016) 4118-24.

[59] F. Spaepen, A microscopic mechanism for steady state inhomogeneous flow in metallic glasses, Acta metallurgica 25(4) (1977) 407-415.

[60] A.V. Khomich, R.A. Khmelnitskiy, V.A. Dravin, A.A. Gippius, E.V. Zavedeev, I.I. Vlasov, Radiation damage in diamonds subjected to helium implantation, Physics of the Solid State 49(9) (2007) 1661-1665. 
